\title{
A 10-year-old Child with Severe Subglottic Stenosis Following Intubation: A Case Report
}

\author{
Venus Chegini $^{1}$ (D),Victoria Chegini2 ${ }^{2 *}$ (D), Mahdis Esfahani ${ }^{3}$ (D) Hossein $^{\text {Moeini }}{ }^{4}$ (D)
}

1. Department of Laparoscopic Obstetrics and Gynecology, Faculty of Medicine, Qazvin University of Medical Sciences, Qazvin, Iran

2. Department of Pediatrics, School of Medicine, Shahid Beheshti University of Medical Sciences, Tehran, Iran.

3. Clinical Research Development Unit, Qods Hospital, Qazvin University of Medical Sciences, Qazvin, Iran.

4. Department of Pediatric Anesthesia, Children Growth Research Center, Research Institute for Prevention of Non-Communicable Diseases, Qazvin

University of Medical Sciences, Qazvin, Iran.

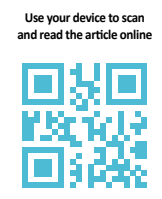

Crtation Chegini V, Chegini V, Esfahani M, Moeini H. A 10-year-old Child with Severe Subglottic Stenosis Following Intubation: A Case Report . J Inflamm Dis. 2021; 25(1):39-44. http://dx.doi.org/10.32598/JQUMS.25.1.4

doij http://dx.doi.org/10.32598/JQUMS.25.1.4

(c) (1) (8)

Article info:

Received: 17 May 2020

Accepted: 12 Feb 2021

Publish: 01 Apr 2021

Keywords:

Subglottic stenosis,

Children, Intensive care,

Intubation, Bronchoscopy

\section{ABSTRACT}

Background: Subglottic stenosis is among the most common airway problems in children, i.e., acquired or congenital. More than $90 \%$ of acquired cases are secondary to endotracheal intubation and occur iatrogenically. Subglottic stenosis is an unexpected problem that requires timely diagnosis and intervention. Most cases of subglottic stenosis in children are mild to moderate.

Case Presentation: The presented patient was a 10-year-old child who, after long intubation due to head trauma following early discharge from the surgical center, was referred to the Children's Hospital on the same day with a complaint of high fever and shortness of breath. With the development of respiratory distress and cyanosis, the patient was transferred to the intensive care unit. Due to the impossibility of passing the tracheal tube with the appropriate age for the patient, the initial diagnosis of subglottic stenosis was established and a tracheostomy was performed. Diagnostic bronchoscopy confirmed severe subglottic stenosis, and the child was referred to a specialized Ear, Nose, Throat (ENT) center for the repair of the tracheal stenosis.

Conclusion: One of the most common causes of stridor in children is subglottic stenosis following prolonged intubation. Recognizing the predisposing factors, prevention, strong clinical suspicion, timely diagnosis, and treatment can prevent further adverse complications or consequences in children.

\section{"Corresponding Author:}

Victoria Chegini

Address: Department of Pediatrics, School of Medicine, Shahid Beheshti University of Medical Sciences, Tehran, Iran.

Phone: +98 (28) 33328709

E-mail: victoria_che@yahoo.com 


\section{Introduction}

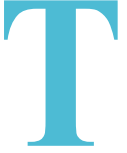

racheal subglottic stenosis is among the airway problems in infants and children, i.e., observed in two types of congenital or acquired. The subglottis of the anatomical area of the trachea is just below the surface of the vocal cords and adjacent to the cricoid cartilage; it is the narrowest part of the airway in infants and children. Subglottic stenosis involves reducing the diameter of the airway in this area and can be life-threatening due to the inflexibility of this region. Besides, $90 \%$ of cases of acquired subglottic stenosis follow iatrogenic endotracheal intubation [1]. According to retrospective or prospective assessments, the prevalence of subglottic stenosis in children ranges from $0.6 \%$ to $11.38 \%[2,3]$.

The most common risk factors for subglottic stenosis, especially in children, include prolonged intubation; the improper selection of endotracheal tube size and inadequate sedation and analgesia; repetitive intubation, and nursing errors, like traumatic suctioning. Other predisposing factors for subglottic stenosis consist of the underlying diseases of the respiratory system, Gastroesophageal Reflux Disease (GERD), premature birth, brain injury, congenital airway disorders, cerebral palsy, Down syndrome, prolonged seizures, viral infection leading to intubation, the inadequate perfusion of the airway mucosa due to hypotension, anemia, sepsis, obesity, diabetes, and shock [4-10].

According to the modified Cotton-Myer classification, the extent of subglottic stenosis is divided into four grades (degrees) and each grade is divided into 3 subclasses [11] (Figure 1):

The first sign of subglottic stenosis is stridor at the time of inspiration. Furthermore, if obstruction progresses, biphasic stridor occurs. Stridor after extubation is a frequent complication in children with a prevalence of $44 \%$ and severe, late, or progressive cases; this condition requires immediate examination and treatment [12].

The gold standard for diagnosing subglottic stenosis is laryngobronchoscopy. Mild to moderate cases require medical treatment or bronchoscopic intervention. Moreover, moderate to severe cases require open surgery and tracheostomy [4].

Most cases of subglottic stenosis in children are moderate and mild (grades $1 \& 2$ ) and often require no surgical repair [13]. In this report, we presented a child with severe subglottic stenosis who required extensive resection surgery. Considering the consequences of this complication in children, it is critical to recognize the risk factors, prevention, timely diagnosis, as well as prompt and appropriate intervention.

\section{Case Presentation}

A 10-year-old boy was referred to a surgical hospital following a car accident. is the patient was immediately intubated in the emergency room and transferred to the Intensive Care Unit (ICU) due to severe head trauma, cerebral hemorrhage, and the loss of consciousness. The child underwent mechanical ventilation for 28 days. Despite the long-term intubation, he was discharged home one day after extubation with Glasgow Coma Scale (GCS)=13-14 and paraplegia.

The reported patient was admitted 12 hours after discharge due to high fever, respiratory distress, and the vital signs of temprature $=39^{\circ} \mathrm{C}$, tachypnea (Pulse Rate $(P R)=42 / \mathrm{min})$, tachycardia $(\mathrm{PR}=138 / \mathrm{min})$, accordingly, he was prescribed adjuvant oxygen and broad-spectrum antibiotics. The patient developed respiratory distress and stridor one day after admission; he responded partially and temporarily to pharmacotherapy; however, after a few hours, he again developed progressive and severe respiratory distress, followed by cyanosis, seizures, and the loss of consciousness. Additionally, he presented the vital signs of $\mathrm{GCS}=6$ and $\mathrm{PCO}_{2}=80 \mathrm{mmHg}, \mathrm{PR}=68 /$ $\min , \mathrm{SPO}_{2}=82 \%$ by receiving oxygen through a high flow face mask transferred to pediatric ICU. Rapid Sequence Intubation (RSI) was performed immediately. The intubation of the patient was not possible with cuffed Endotracheal Tube (ETT) numbers 6 and 5.5 and the child were intubated with ETT number 5 . The next day, due to the obstruction of the ETT with secretions and the lack of proper ventilation, the reported patient was reintubated. This time, it was impossible to pass the ETT with the previous size; thus, the patient was intubated with ETT number 4 using a guidewire. Consequently, the diagnosis of subglottic stenosis was established, and a tracheostomy was inserted for the patient. After the stabilization of respiratory and hemodynamic status, a diagnostic laryngobronchoscopy was performed by an otolaryngologist. In bronchoscopic view, severe subglottic stenosis (grade 3 ) was observed at a distance of $3 \mathrm{~cm}$ from the lower edge of the vocal cord (Figure 2).

A neck and chest Computer Tomography (CT) scan presented subglottic stenosis without other airway abnormalities. After weaning, the child was referred to a specialized Ear, Nose, Throat (ENT) center to repair the stenosis of the trachea. The child underwent direct 


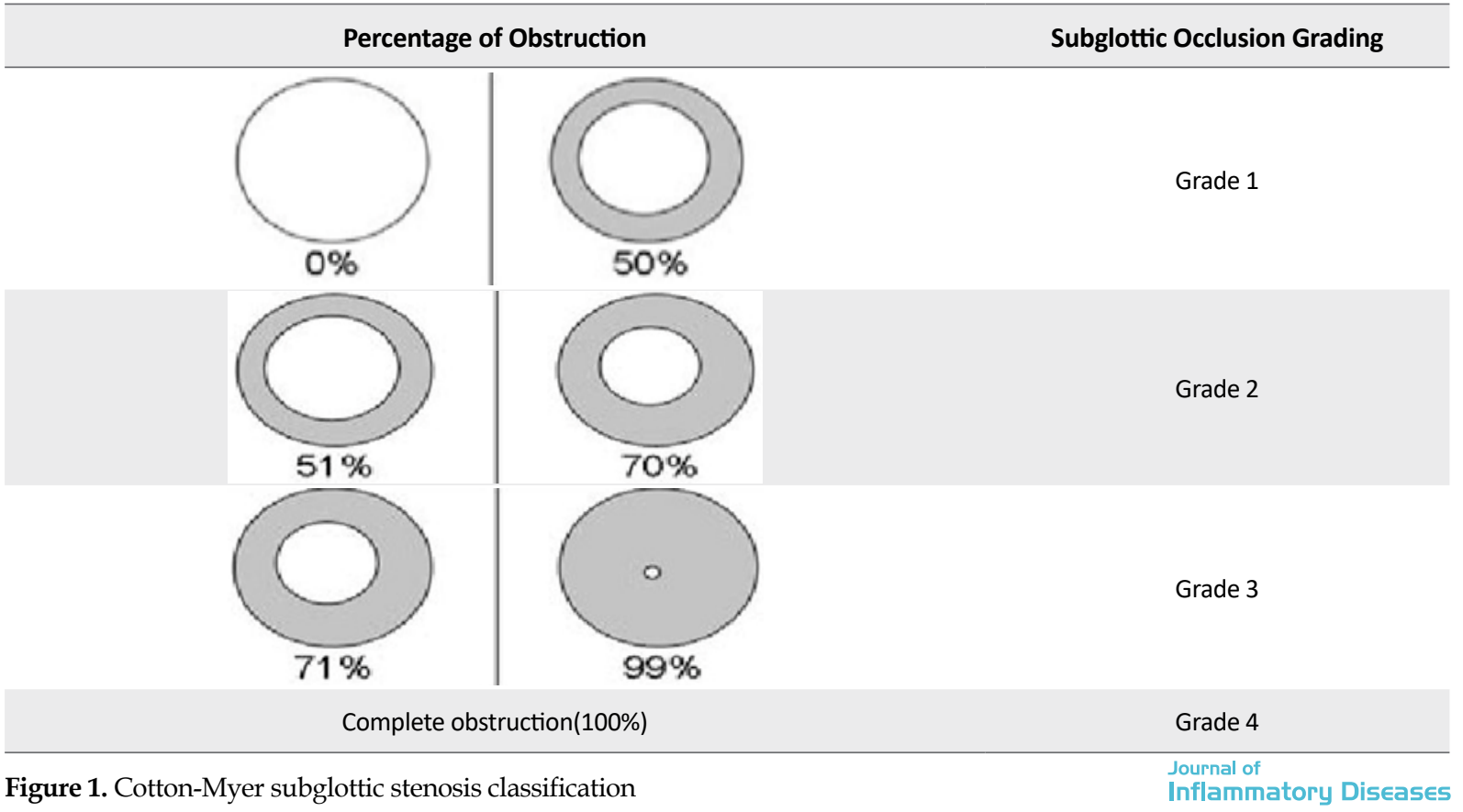

Grade 1: Obstruction of $<50 \%$ of the lumen diameter

Grade 2: Obstruction of $50 \%-70 \%$ of the lumen diameter

Grade 3: Obstruction of 70\%-99\% of the lumen diameter

Grade 4: Complete occlusion

diagnostic laryngobronchoscopy. Moreover, completed tracheal stenosis (grade 4) was detected at a distance of $3-4 \mathrm{~cm}$ below the vocal cords. As a result, the cricotracheal resection of the stenosis area with the length of two cartilages up to the carina and end-to-end anastomosis was performed and re-tracheostomy was conducted lower than the previous level. After 6 days, the patient's tracheostomy was removed and the child has verbally and physically returned to a near-normal state and only manifested a slight change in the tone of voice without respiratory problems. The report of this patient was conducted after obtaining the relevant ethical approval (Code: IR.QUMS.REC.1399.170).

\section{Discussion}

Subglottic stenosis following prolonged intubation is the main differential diagnosis of stridor in children. Preventing subglottic stenosis in children due to high morbidity and mortality requires special attention.

Selecting the appropriate size of the tracheal tube, the stability of the patient's hemodynamic status, especially maintaining blood pressure within the normal range for age, the optimal use of adequate sedatives and analgesics even if the level of consciousness is low, the prevention and treatment of GERD, the avoidance of high pressure
Subcategory (a): Subglottic stenosis alone

Subcategory (b): Stenosis comorbid with other diseases

Subcategory (c): Glottis involvement

Subcategory (d): Includes both (b) and (c)

and traumatic suction, fixing the air tube to avoid unexpected extubation, and the need for emergency intubation, using a smaller tube size if intubation needs to be repeated, attempting to extubate as soon as possible, and failing to discharge early after prolonged intubation can greatly reduce the complications of intubation.

Significant progress has been made in treating subglottic stenosis in children; however, its management remains a controversial challenge. Available treatment methods include pharmacotherapy, tracheostomy, balloon endoscopic dilatation, endoscopic posterior cricoid split with cartilage graft, endoscopic anterior cricoid split with balloon dilation, Laryngotracheal Reconstruction (LTR), and partial Cricotracheal Resection (CTR) [1]. Most mild cases are treated with medications, including racemic epinephrine and systemic or inhaled corticosteroids [14]

Balloon endoscopic dilatation, endoscopic posterior cricoid split with cartilage graft, and endoscopic anterior cricoid split with balloon dilation are used in mild to moderate cases. Moreover, moderate to severe cases require endoscopic or open surgical resection and tracheostomy dependence [1] 


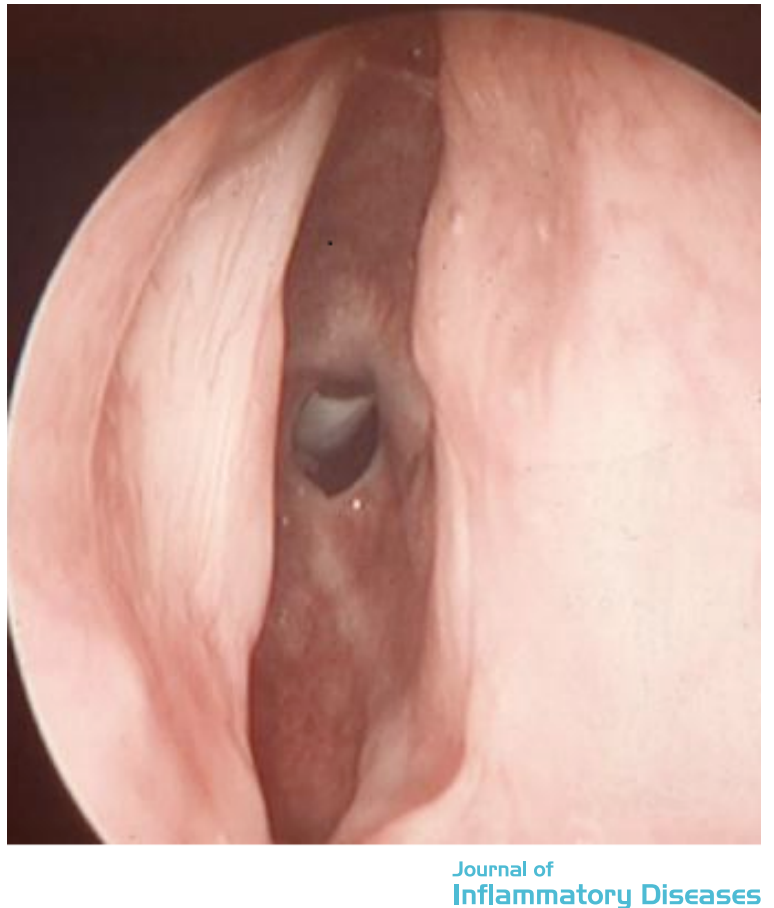

Figure 2. The bronchoscopic index of the reported patient, subglottic stenosis grade 3

According to systematic review studies, endoscopic balloon dilatation has been effective in two-thirds of mild to moderate cases; however, the rate of failure has elevated with the increasing severity of stenosis and intubation period $[15,16]$.

According to a retrospective study, in $84.5 \%$ of patients with moderate to severe post-intubation subglottic stenosis who were treated with one of the laryngotracheal reconstruction, partial cricotracheal resection, and anterior cricoid split methods, only one surgical procedure was required to achieve decannulation [17].

\section{Conclusion}

In the presented case, due to grade 4 subglottic stenosis, other treatment methods were ineffective; therefore, open surgery, including stenosis resection and two-ended anastomosis was performed. Fortunately, the patient's long-term prognosis was satisfactory.

\section{Ethical Considerations}

\section{Compliance with ethical guidelines}

The report of this patient was conducted after obtaining the relevant ethical approval (Code: IR.QUMS. REC.1399.170).

\section{Funding}

This research did not receive any grant from funding agencies in the public, commercial, or non-profit sectors.

\section{Authors' contributions}

All authors equally contributed to preparing this article.

\section{Conflict of interest}

The authors declared no conflict of interest.

\section{Acknowledgments}

We thank the patient's family for their cooperation in this study. We also thank the clinical Research Development Unit, Qods Hospital, Qazvin University of Medical Sciences, for all their support and guidance during carrying out this study.

\section{References}

[1] Marston AP, White DR. Subglottic stenosis. Clin Perinatol. 2018; 45(4):787-804. [DOI:10.1016/j.clp.2018.07.013] [PMID]

[2] Rodríguez H, Cuestas G, Botto H, Cocciaglia A, Nieto M, Zanetta A. Post-intubation subglottic stenosis in children. Diagnosis, treatment and prevention of moderate and severe stenosis. Acta Otorrinolaringol. 2013; 64(5):339-44. [DOI:10.1016/j.otoeng.2013.10.005] [PMID]

[3] Schweiger C, Marostica PJ, Smith MM, Manica D, Carvalho PR, Kuhl G. Incidence of post-intubation subglottic stenosis in children: Prospective study. J Laryngol Otol. 2013; 127(4):399403. [DOI:10.1017/S002221511300025X] [PMID]

[4] Rutter M, Kuo IC, Predicting and managing the development of subglottic stenosis following intubation in children. J Pediatr. 2020; 96(1):1-3. [DOI:10.1016/j.jped.2019.04.001] [PMID]

[5] Wei JL, Bond J. Management and prevention of endotracheal intubation injury in neonates. Curr Opin Otolaryngol Head Neck Surg. 2011; 19(6):474-7. [DOI:10.1097/ MOO.0b013e32834c7b5c] [PMID]

[6] Poetker DM, Ettema SL, Blumin JH, Toohill RJ, Merati AL. Association of airway abnormalities and risk factors in 37 subglottic stenosis patients. Otolaryngol Head Neck Otolaryngol Head Neck Surg. 2006; 135(3):434-7. [DOI:10.1016/j. otohns.2006.04.013] [PMID]

[7] Schweiger C, Manica D, Pereira DRR, Carvalho PRA, Piva JP, Kuhl G, et al. Undersedation is a risk factor for the development of subglottic stenosis in intubated children. J Pediatr. 2017; 93(4):351-5. [DOI:10.1016/j.jpedp.2017.02.004] [PMID]

[8] Manica D, Schweiger C, Maróstica PJC, Kuhl G, Carvalho PRA. Association between length of intubation and subglot- 
tic stenosis in children. Laryngoscope. 2013; 123(4):1049-54. [DOI:10.1002/lary.23771] [PMID]

[9] Jefferson ND, Cohen AP, Rutter MJ. Subglottic stenosis Semin Pediatr Surg. 2016; 25(3):138-43. [DOI:10.1053/j.sempedsurg.2016.02.006] [PMID]

[10] Nicolli EA, Carey RM, Farquhar D, Haft S, Alfonso KP, Mirza N. Risk factors for adult acquired subglottic stenosis. J Laryngol Otol. 2017; 131(3):264-7. [DOI: 10.1017/ S0022215116009798] [PMID]

[11] Monnier P. Acquired post-intubation and tracheostomyrelated stenoses. In: Monnier P, editor. Pediatric Airway Surgery. Berlin/Heidelberg: Springer; 2011. pp. 183-98. [DOI:10.1007/978-3-642-13535-4_14]

[12] Schweiger C, Eneas LV, Manica D, de Souza Saleh Netto C, Carvalho PRA, Piva JP, et al. Accuracy of stridor-based diagnosis of post-intubation subglottic stenosis in pediatric patients. J Pediatr. 2020; 96(1):39-45. [DOI:10.1016/j. jped.2018.08.004] [PMID]

[13] McClay JE. Subglottic stenosis in children [Internet]. 2021 [Updated 2021 February 22]. Available from: https://emedicine.medscape.com/article/864208-overview

[14] Sinha A, Jayashree M, Singhi S. Aerosolized L-epinephrine vs budesonide for post extubation stridor: A randomized controlled trial. Indian Pediatr. 2010; 47(4):317-22. [DOI:10.1007/ s13312-010-0060-z] [PMID]

[15] Lang M, Brietzke SE. A systematic review and meta-analysis of endoscopic balloon dilation of pediatric subglottic stenosis. Otolaryngol Head Neck Surg. 2014; 150(2):174-9. [DOI:10.1177/0194599813510867] [PMID]

[16] Romero Manteola EJ, Patiño González C, Ravetta P, Defago $\mathrm{V}$, Tessi C. Dilation with rigid dilators as primary treatment of subglottic stenosis in pediatrics. Pulmonology. 2020; S25310437(20)30046-5. [DOI:10.1016/j.pulmoe.2020.03.007] [PMID]

[17] Bitar MA, Al Barazi R, Barakeh R. Airway reconstruction: Review of an approach to the advanced-stage laryngotracheal stenosis. Braz J Otorhinolaryngol. 2017; 83(3):299-312. [DOI:10.1016/j.bjorl.2016.03.012] [PMID] 
This Page Intentionally Left Blank 